\title{
T Wave Amplitude Deviation as a Participation Marker of Glycolysis in Myocardial Repolarization
}

\author{
Mikhail U. Alekseev1,2, Aleksandr M. Alekseev² \\ ${ }^{1} J u v e n t a$ Diagnostic Center, Saint Petersburg, Russia \\ ${ }^{2}$ Tozka Diagnostics LLC, Moscow, Russia \\ Email: michaelalekseyev@gmail.com
}

How to cite this paper: Alekseev, M.U. and Alekseev, A.M. (2019) T Wave Amplitude Deviation as a Participation Marker of Glycolysis in Myocardial Repolarization. World Journal of Cardiovascular Diseases, 9, 343-353.

https://doi.org/10.4236/wjcd.2019.95030

Received: April 7, 2019

Accepted: May 18, 2019

Published: May 21, 2019

Copyright $\odot 2019$ by author(s) and Scientific Research Publishing Inc. This work is licensed under the Creative Commons Attribution-NonCommercial International License (CC BY-NC 4.0). http://creativecommons.org/licenses/by-nc/4.0/

\begin{abstract}
Glucose metabolism is essential for heart physiology, especially in ischemic conditions. Anaerobic glycolysis participates along with aerobic fatty acid oxidation in generating energy supply, and the balance is shifted towards fatty acids. In the case of aerobic path restriction due to coronary artery disease (CAD), this ratio changes. The aim of the present research was to discover the aspects of glycolysis in cardiac electrophysiology. We utilized the normalized (by average value) $\mathrm{T}$ wave amplitude deviation values, named the $\mathrm{G}$ criterion, from high-resolution, 12-channel, 120-seconds-long electrocardiograms, which were obtained using DACG technology. The calculated G criterion allows a quantitative assessment of the ischemic process and corresponds to the functional class (FC) according to the Canadian cardiovascular classification. We examined 22 healthy people and 79 proven CAD patients: 24 II FC, 40 III FC and 15 IV FC by original ECG and fasting blood sugar tests. A strong correlation was found between the average $G$ criterion from all 12 channels and the level of fasting blood sugar $(\mathrm{r}=+0.88)$. In the control group, blood sugar levels were $91 \pm 6.5 \mathrm{mg} / \mathrm{dl}$, II FC $103 \pm 14.8 \mathrm{mg} / \mathrm{dl}(\mathrm{p}<0.01)$, III FC $113 \pm 23.4$ $\mathrm{mg} / \mathrm{dl}(\mathrm{p}<0.001)$, and IV FC $152 \pm 42.4(\mathrm{p}<0.001)$. Moreover, the correlation factor (average $\mathrm{G}$ criterion and blood sugar) was strong and positive in each group: control +0.72 , II FC +0.83 , III FC +0.76 , and IV FC +0.89 . For the first time, heart electrical activity from the ECG curve was associated with high glucose level as one of the main CAD risk factors. The presence of such dependence of increased blood sugar level from the severity of the ischemic process in the myocardium indicates that hyperglycemic reaction is adaptive and can accelerate the anaerobic glycolysis for energy demand compensation in heart muscle.
\end{abstract}




\section{Keywords}

Electrocardiogram, T Wave Deviation, Blood Sugar, Hyperglycemia, Glycolysis

\section{Introduction}

Coronary artery disease (CAD), also known as ischemic heart disease (IHD), is a condition in which there is an inadequate supply of blood and oxygen to a portion of the myocardium. It results in higher morbidity, mortality, and medical costs than any other illness in the developed world. Though it may affect individuals at any age, it is much more common in older ages. The risk approximately triples with each decade of life.

IHD typically occurs when there is an imbalance between energy supply and demand. The most common cause of myocardial ischemia is an atherosclerotic lesion of the epicardial coronary arteries sufficient to cause a regional reduction in myocardial blood flow and inadequate perfusion of the myocardium supplied by the involved coronary artery.

Adenosine triphosphate (ATP) is the main transporter of chemical energy, and cardiac muscle can use a variety of substrates to oxidatively regenerate ATP depending on the availability: mostly fatty acids, glucose, lactate, and ketone bodies; amino acids are rarely used [1]. The substrate availability is created by different metabolic environments such as fasting, feeding, exercise, various of neurohumoral factors [2] [3] [4].

Early studies in animals and human [3] [4] showed that after an overnight fast, the heart extracts free fatty acids (FFA), lactate, and glucose from the blood. If one assumes complete oxidation of extracted substrates, then fatty acids are the major oxidative fuel for the heart ( $60 \%-100 \%$ of the oxygen consumption) with a lesser contribution from lactate and glucose ( $0 \%-20 \%$ from each) [3] [5] [6]. During exercise, the predominant substrate of heart energy is lactate because lactic acid plasma concentrations significantly increase and replaces the glucose from metabolism circle [7] [8].

Ischemic myocardium in the low oxygen conditions can utilize the carbohydrates including glycogen from glucose for anaerobic ATP supply with production of lactic acid in the cells. These metabolic changes force the myocardial cells to increase their glucose uptake leading to glycogenolysis and glycolytic flux [9] [10]. In moderate ischemia (reduction of coronary flow by 75\%), glucose uptake remains unchanged, while glucose extraction increases and metabolism of glucose is directed from oxidation to lactate production [11].

In severe ischemia, myocardial glucose extraction is inversely related to coronary flow until the degree of ischemia becomes so severe that glycolysis is inhibited by the accumulation of its products [12] [13]. Glycolysis is partially inhibited, and glucose uptake progressively decreases. Protons, $\mathrm{Na}^{+}$and $\mathrm{Ca}^{2+}$ continue accumulating. The decline of glucose uptake during prolonged severe 
ischemia may be attenuated by various interventions protecting the heart against ischemic injury such as an increase in the extracellular glucose concentration or addition of insulin [14] [15] [16] [17] [18]. These interventions promote glucose uptake to meet the increased demand for glucose moieties as an energy source.

The Canadian Cardiovascular Society grading of angina pectoris allows for quantitative and nearly precise assessment of the severity of angina pectoris. The limitation of this classification is the subjectivity of a patient's complaints, which depend on the individual's sensitivity. In previous studies we discovered correlation between normalized T-wave amplitude deviation (NTWaD), i.e., the G-criterion, and the functional classes according the Canadian Cardiovascular Society. Dividing the maximum and minimum values of NTWaD or G-criterion in ischemic and normal zones of the heart can quantitatively assess the severity of the ischemic process in myocardium regardless of the patient's sensitivity and complaints. Other work with nitrate administration proved our theory-G-criterion reflects the ischemic status of myocardium and is useful in drug efficacy administration control in real-time [19] [20].

In another work, we showed that differences between normalized T-wave time deviation values (NTWtD) from different ECG channels can detect coronary artery disease with high accuracy including the approximate percentage of lesions and their localization. This finding explains why $\mathrm{T}$ wave time reflects an aerobic process in the myocardium. They are disturbed by ischemia with an accumulation of protons, reduction of intracellular $\mathrm{pH}$, and increased lactate production. The maximum concentration of these metabolites injures myocardial cells and causes necrosis. Moreover, the maximum values of NTWtD corresponded to acute myocardial infarction development in the nearest $24-48$ hours. These findings encourage us to explore the metabolic processes that underlie T-wave and $\mathrm{T}$-wave deviation.

\section{Methods}

We used the technology Digital Analytic Cardiography (DACG) that was based on in-depth analysis of temporal and amplitude parameters of well-known electrocardiography (ECG) parameters [19]. The 12-channel usual electrocardiograph Amedtec Cardio Part 12 USB in rhythm mode was used for this goal. The fundamental differences and common ECG device are as follows:

The resolution of analog to digital converter of ECG device with sample rate $8000 \mathrm{~Hz}[125 \mu \mathrm{s}]$ in time and resolution $1 \mu \mathrm{V} / \mathrm{LSB}$ in amplitude measurements. ECG baseline wandering, electromagnetic power line interference and muscle trembling was cancelled by $0.5 \mathrm{~Hz}, 50 \mathrm{~Hz}$ and $35 \mathrm{~Hz}$ (low pass) filters, as the aim of our work was to characterize a deviation values, not absolute ones.

After adaptation in the lying position, disposable contact silver/silver chloride electrodes were attached to each patient: four limb leads and six precordial leads. The recording was performed in all the 12 channels simultaneously for 240 seconds as required for $200 \mathrm{PQRST}$-complexes. The data were then transferred from the ECG device to a computer. The ECG recordings were processed with 
the manufacturer's software to calculate the $\mathrm{G}$ and $\mathrm{L}$ criteria.

It is known that the first ischemic changes are represented on the surface ECG by $\mathrm{T}$ wave abnormalities (hyper-acute, inverted, biphasic and flat) and ST-segment changes (elevation or depression). F calculate the G-criterion, we use the T-wave amplitude from each of the 12 channels separately. T wave amplitudes for each heartbeat were assessed from the baseline taken from PR segment level.

We characterized the $200 \mathrm{~T}$-wave amplitudes with standard deviation and average values (Figure 1). To calculation the G-criterion, we relate the standard deviation of $200 \mathrm{~T}$-wave amplitudes (Twa) to the average value. For convenience of result presentation, we reduce the values to one. We then compared the $G$ criteria from each channel, which reflects the different parts of the heart. The differences in criteria between channels gives us quantitative features of the ischemic zones and non-ischemic zones in the myocardium. We then relate the maximum or ischemic value of the G-criterion from one channel to the minimum or non-ischemic value of the G-criterion from another channel to calculate the second order G-criterion (SOGdiff criterion).

It is known that glycolysis plays a crucial role in cardiac metabolism-especially in ischemic conditions; thus, we decided to first compare fasting glycaemia level with SOGdiff criterion. CAD often affects vast amounts of cardiac muscle, and it is logical to include the SOG criterion for all available $\mathrm{G}$ criterion values from all channels. Consequently, we calculated average value from $\mathrm{G}$ criterion values from one record and named them the averaged SOG criterion (SOGave criterion).

Glucose in blood is the most frequent analyzed parameter in a clinical chemistry laboratory. The fasting blood sugar test was made in the morning before breakfast in all cases. It was recommended not to eat or drink except water for at least 8 hours before having a blood glucose test. Later, for patients with diabetes, glucose levels are often checked both while fasting and after meals to reveal blood glucose curves and corrections. Here, we utilized only fasting blood level (in $\mathrm{mmol} / \mathrm{l}$ ) assessed by hexokinase kits.

\section{Materials}

This study examined four groups of patients: The first group included 22 young healthy people aged $34 \pm 9.3$. The second group includes 24 patients aged $50.8 \pm$ 8.2 with II FC, 40 patients aged $58.1 \pm 7.7$ with III FC, and 15 patients aged 64.9 \pm 10.2 with IV FC. The diagnosis was confirmed in all 79 cases by resting ECG examination, stress testing, and (in 63 cases) by invasive coronary angiography (moderate CAD-19 and severe CAD-44 patients). Starting from the special DACG examination and fasting blood sugar testing, all patients from II, III, and IV FC administrated antianginal and hypotensive therapy. Of the 79 patients, only 8 took hypoglycemic and insulin treatment because the blood sugar tests were made before blood sugar analyses.

In current study, we did not include patients with atrial fibrillation, atrial flut- 
tering because permanent $\mathrm{f}$ and $\mathrm{F}$ waves from chaotic atrial electrical activity seriously disturbs the baseline and original $\mathrm{T}$ wave deviation.

\section{Statistical Analysis}

STATISTICA 12.6 was used for statistical analyses. The data are presented as absolute values of criteria or mean, standard deviation, SD. Unpaired Student's t test was used for comparisons. A probability value of $\mathrm{P}<0.05$ was considered statistically significant. Confidence interval (CI \%) was also computed from the statistics of the observed data. The $95 \%$ confidence level was used. The correlation coefficient $(r)$ was measured as a linear association between tested values and the calculated criterion. A correlation coefficient of $r=+1$ indicates that the two variables are perfectly related in a positive linear sense, while a correlation coefficient $r=0$ indicates that there is no linear relationship between the two variables.

\section{Results}

Table 1 summarizes the various parameters of SOGave, SOGdiff criteria, blood glucose tests, and significance of differences among functional classes. The difference of criteria and glucose level among functional groups was significant ( $\mathrm{p}$ $<0.05)$, excluding blood glucose in II FC (5.43 $\pm 0.7 ; 95 \%$ CI $4.7-6.16)$ and III FC $(5.97 \pm 1.05 ; 95 \%$ CI $4.92-7.02)$ with $\mathrm{p}=0.058$.

Table 1. Result presented as means \pm SD. Fasting blood sugar level (Fasting BS; milligram/ decilitre), normalized $\mathrm{T}$ wave amplitude deviation ( $\mathrm{G}$ criterion, percent), difference between $\mathrm{G}$ criteria in ischemic and normal zone (SOGdiff), average value of all G criteria from all available ECG channels (SOGave, percentage). Mean difference between II FC* and normal; III FC* and normal, II FC; IV FC and normal, II FC, III FC are indicated: ns $=$ not significant; ${ }^{*} \mathrm{P}<0.05 ;{ }^{\dagger} \mathrm{P}<0.01 ;{ }^{\ddagger} \mathrm{P}<0.001$.

\begin{tabular}{|c|c|c|c|c|}
\hline & \multicolumn{4}{|c|}{ Functional classes according CCS } \\
\hline & Normal $(\mathrm{n}=22)$ & $\mathrm{II}(\mathrm{n}=24)$ & $\mathrm{III}(\mathrm{n}=40)$ & IV $(n=15)$ \\
\hline Age & $34 \pm 9.3$ & $50.8 \pm 8.2$ & $58.1 \pm 7.7$ & $64.9 \pm 10.2$ \\
\hline Male/Female & $9 / 11$ & $14 / 10$ & $28 / 12$ & $8 / 7$ \\
\hline \multicolumn{5}{|c|}{ Blood sugar } \\
\hline Fasting BS (mg/dl) & $91 \pm 6.5$ & $103 \pm 14.8^{\dagger}$ & $113 \pm 23.4^{\ddagger, n s}$ & $152 \pm 42.5^{\ddagger \neq,+\neq}$ \\
\hline \multicolumn{5}{|c|}{ G criterion } \\
\hline SOGdiff & $1.64 \pm 0.33$ & $2.29 \pm 0.44^{\ddagger}$ & $3.71 \pm 0.81^{\ddagger \neq \neq}$ & $5.93 \pm 1.39^{\ddagger \neq, \neq \neq}$ \\
\hline $95 \%[\mathrm{CI}]$ & $1.5-1.79$ & $2.12-2.47$ & $3.45-3.96$ & $5.23-6.64$ \\
\hline SOGave & $5.11 \pm 0.62$ & $5.88 \pm 0.81^{\dagger}$ & $6.65 \pm 1.52^{\ddagger, \star}$ & $8.34 \pm 2.35^{\ddagger, \ldots, \dagger}$ \\
\hline $95 \%[\mathrm{CI}]$ & $4.84-5.38$ & $5.56-6.21$ & $6.18-7.12$ & $7.15-9.52$ \\
\hline \multicolumn{5}{|l|}{ Correlation factor } \\
\hline SOGdiff/fasting BS (r) & +0.35 & -0.29 & +0.05 & -0.18 \\
\hline SOGave/fasting BS (r) & +0.72 & +0.83 & +0.76 & +0.89 \\
\hline
\end{tabular}


Both SOGdiff and SOGave criteria were analyzed separately. In our previous articles, we proved the dependence of SOGdiff from functional classes according to CCS. However, the correlation of SOGdiff and blood glucose turned out to be weak $(r=+0.17)$ in all examined cases. The utilization of SOGave was alternative data and corroborated our theory (Figure 2).

Strong correlation $(\mathrm{r}=+0.88)$ between SOGave criterion and blood glucose level was found. Figure 2 shows the level of correlation between blood sugar level SOGave criterion in all groups with nearly stable variance of values in all groups. The strong correlation was found in the control or normal group ( $\mathrm{r}=$ +0.72 ). Direct proportional communication was traced between glucose level and SOGave criterion against the background of CAD: in II FC r $=+0.83$, III FC $\mathrm{r}=+0.76$ and IV FC $\mathrm{r}=+0.89$.

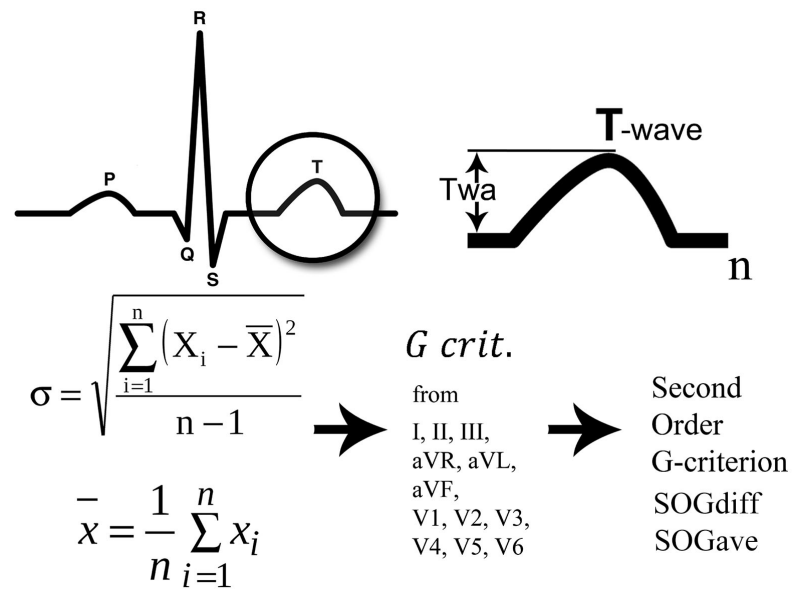

Figure 1. The algorithm of G-criterion calculation, where $\mathrm{n}$ means the number of T-wave amplitude measurements in 60 seconds; Twa$\mathrm{T}$-wave amplitudes; $\sigma$-standard deviation value; $\mathrm{x}$-average value.

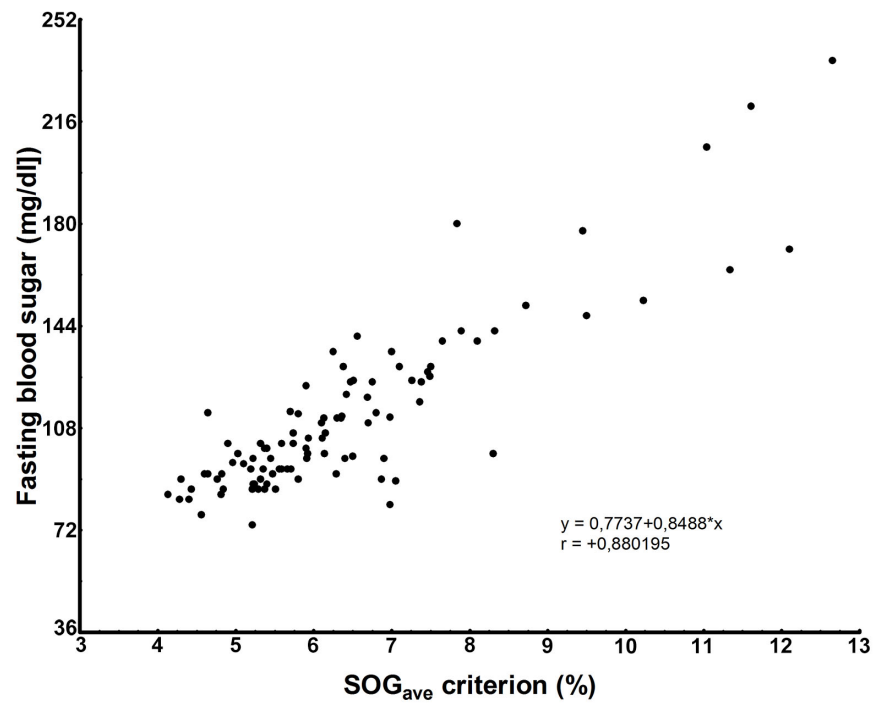

Figure 2. The correlation between SOGave (\%) criterion and fasting blood sugar level (mg/dl). 


\section{Discussion}

T-wave amplitude deviation can assess the degree of repolarization process in both normal and in ischemic myocardium in each channel. Such pathological processes are usually evoked in with insufficient blood flow or oxygen supply for aerobic production of ATP in cardiomyocytes. This results in ATP compensation by anaerobic pathways including anaerobic glycolysis, but this is quite limited [21] [22]. In normal or non-ischemic myocardium, the energy produced by fatty acids accounts for approximately $70 \%$ of the energy with glucose accounting for 30\%; this ratio changes in ischemic conditions [8] [21]-[26].

Moderate ischemia is a more oxygen-intensive but more efficient approach to fatty acid oxidation. It continues to dominate cardiomyocytes that reflect in beat-to-beat instability of ATP formation [7] [22] [27] [28]. As the depth of ischemia increases, it can steadily inhibit the aerobic pathways of energy supply that occur along with a gradual enhancement of anaerobic methods of ATP formationfrom anaerobic glycolysis [21] [22]. The main problem in anaerobic conditionsis utilization derangement and accumulation of fatty acids and anaerobic glycose metabolism products including lactate with gradual acidosis enhancement in cardiac cells. The consequence of these processes is increased instability of ATP formation.

The resting potential of the cell and relatively stable ions maintenance is accomplished via metabolic changes in ischemic myocardium, changes mainly in $\mathrm{K}$ (ATP)-channel activity, $\mathrm{Na}^{+} / \mathrm{K}^{+}$ATPase and $\mathrm{Na}^{+} / \mathrm{H}^{+}$exchanger with linked $\mathrm{Na}^{+} / \mathrm{Ca}^{2+}$ exchanger, $\mathrm{Ca}^{2+}$ activated potassium channels.

Potassium channels are very sensitive to the level of ATP production-especially ATP-dependent $\mathrm{K}^{+}$channels. In normal, non-ischemicmyocardium, the high level of ATP is constantly maintained, and these channels remain closed. The channel opens in cases of overloading of the heart, hypoxia, or ischemia. This causes additional $\mathrm{K}^{+}$current from the cell. The blockade of K (ATP) channels with a high intracellular concentration of ATP prevents the development of metabolic adaptation of the myocardium to ischemia [29]. This worsens the functional parameters of the cardiovascular systemand leads to greater cardiac ischemic damage.

However, in case of decreasing of intracellular ATP concentration, the activation of K(ATP) channelspromotes the metabolic adaptation of myocardium to ischemia. This process causes a slight mechanical activity reduction of cardiomyocytes due to lowered action potential duration [30]. Consequently, in myocardium, the bond between metabolic instability-including the instability of the formation of ATP and K (ATP) channels activity-and repolarization processes is obvious.

In addition to $\mathrm{K}$ (ATP) channels, $\mathrm{Ca}^{2+}$ sensitive potassium channels also play a significant role in the repolarization process and can be strongly affected by ischemic process-especially when ischemia-induced fall in intracellular $\mathrm{pH}$ is reached [31] [32]. It results in greater amount of ATP required by sarcoplasmic 
$\mathrm{Ca}^{2+}$ and higher cytosolic $\mathrm{Ca}^{2+}$ concentration for force generation.

The T-wave is an important element of the ECG. It mostly reflects the process of myocardial repolarization. The electrocardiographic signal allows one indirectly assessing the features of transmembrane currents, and thus the $\mathrm{T}$ wave amplitude deviation reflects a feature of stability or instability in potassium currents. The correlation between the glycemia level and the average value of T-wave amplitude deviation values in all channels shown here suggests that anaerobic glycolysis is an important compensatory mechanism to eliminate the imbalance of ATP production in ischemic myocardium.

The experimental data, published by Noakes demonstrated that glycolysis intensity is strongly associated with the speed of diastolic heart relaxation [33]. Additionally, in ischemic conditions there is an association between the glycolysis intensity and $\mathrm{Ca}^{2+}$ absorption by sarcoplasmic reticulum [34] [35]. Authentic correlation between the blood glucose and $\mathrm{T}$ wave deviation values shows the relation of myocardial repolarization activity from carbohydrate metabolism. It is conspicuous the presence of this correlation as in control group as CAD patients. Moreover, correlation increases along with the depth of ischemic process in myocardium.

A difficult task facing the practitioner is how to evaluate the elevated blood glucose level in CAD patients. If cases of diabetes mellitus, it is absolutely necessary to normalize carbohydrate metabolism. But if this increase glycaemia is a compensatory reaction of the organism to preserve the level of ATP production, then decreased blood sugar complicates the anti-ischemic therapy. It is known that approximately $21 \%$ of CAD patients are asymptomatic [36]. In these cases, if patient has high blood sugar level without clinical presentation of myocardial ischemia, it is necessary to exclude CAD along with carbohydrate metabolism disturbance or possible diabetes mellitus type 2 .

\section{Conclusion}

We show here for the first time a strong correlation between blood sugar level and the ECG criterion. The $\mathrm{G}$ criterion shows the instability of the repolarization process, and this relationship allows us to judge hyperglycemia as a risk factor and compensation process in ischemic conditions. Myocardial glucose metabolism depends not only on the deepness of the ischemic process but also on theoverall heart volume mass involved in ischemic process. The experimental data and high correlation of blood glucose with $\mathrm{T}$ wave deviation pattern, especially in normal group, allows us to presuppose that glycolysis exactly plays a crucial role in heart repolarization and influence on the speed of relaxation process both in healthy and CAD cases.

\section{Conflicts of Interest}

The authors declare no conflicts of interest regarding the publication of this paper. 


\section{References}

[1] Taegtmeyer, H. (1994) Energy Metabolism of the Heart: From Basic Concepts to Clinical Applications. Current Problems in Cardiology, 19, 87-113. https://doi.org/10.1016/0146-2806(94)90008-6

[2] Taegtmeyer, H., Hems, R. and Krebs, H.A. (1980) Utilization of Energy-Providing Substrates in the Isolated Working Rat Heart. Biochemical Journal, 186, 701-711. https://doi.org/10.1042/bj1860701

[3] Randle, P.J., Garland, P.B., Hales, C.N. and Newsholme, E.A. (1963) The Glucose Fatty-Acid Cycle. Its Role in Insulin Sensitivity and the Metabolic Disturbances of Diabetes Mellitus. The Lancet, 1, 785-789. https://doi.org/10.1016/S0140-6736(63)91500-9

[4] Hue, L. and Taegtmeyer, H. (2009) The Randle Cycle Revisited: A New Head for an Old Hat. American Journal of Physiology-Endocrinology and Metabolism, 297, E578-E591. https://doi.org/10.1152/ajpendo.00093.2009

[5] Lopaschuk, G.D. and Saddik, M. (1992) The Relative Contribution of Glucose and Fatty Acids to ATP Production in Hearts Reperfused Following Ischemia. Molecular and Cellular Biochemistry, 116, 111-116. https://doi.org/10.1007/978-1-4615-3514-0_16

[6] Lopaschuk, G.D., Belke, D.D., Gamble, J., Itoi, T. and Schonekess, B.O. (1994) Regulation of Fatty Acid Oxidation in the Mammalian Heart in Health and Disease. Biochimica et Biophysica Acta, 1213, 263-276. https://doi.org/10.1016/0005-2760(94)00082-4

[7] Lassers, B.W., Kaijser, L. and Carlson, L.A. (1972) Myocardial Lipid and Carbohydrate Metabolism in Healthy, Fasting Men at Rest: Studies during Continuous Infusion of 3H-Palmitate. European Journal of Clinical Investigation, 2, 348-358. https://doi.org/10.1111/j.1365-2362.1972.tb00661.x

[8] Gertz, E.W., Wisneski, J.A., Stanley, W.C. and Neese, R.A. (1988) Myocardial Substrate Utilization during Exercise in Humans. Journal of Clinical Investigation, 82, 2017-2025. https://doi.org/10.1172/JCI113822

[9] Bing, R.J. (1955) The Metabolism of the Heart. Harvey Lectures, 50, 27-70.

[10] Morgan, H.E., Henderson, M.J., Regen, D.M. and Park, C.R. (1961) Regulation of Glucose Uptake in Muscle, I: The Effects of Insulin and Anoxia on Glucose Transport and Phosphorylation in the Isolated Perfused Heart of Normal Rats. The Journal of Biological Chemistry, 236, 253-261.

[11] Bolukoglu, H., Goodwin, G.W., Guthrie, P.H., Carmical, S.G., Chen, M.T. and Taegtmeyer, H. (1996) Metabolic Fate of Glucose in Reversible Low-Flow Ischemia of the Isolated Working Rat Heart. American Journal of Physiology, 270, H817H826. https://doi.org/10.1152/ajpheart.1996.270.3.H817

[12] Stanley, W.C., Hall, J.L., Stone, C.K. and Hacker, T.A. (1992) Acute Myocardial Ischemia Causes a Transmural Gradient in Glucose Extraction But Not Glucose Uptake. American Journal of Physiology, 262, H91-H96. https://doi.org/10.1152/ajpheart.1992.262.1.H91

[13] Neely, J.R. and Grotyohann, L.W. (1984) Role of Glycolytic Products in Damage to Myocardium: Dissociation of Adenosine Triphosphate Levels and Recovery of Function of Reperfused Canine Myocardium. Circulation Research, 55, 816-824. https://doi.org/10.1161/01.RES.55.6.816

[14] Vanoverschelde, J.L., Janier, M.F., Bakke, J.E., Marshall, D.R. and Bergmann, S.R. (1994) Rate of Glycolysis during Ischemia Determines Extent of Ischemic Injury 
and Functional Recovery after Reperfusion. American Journal of Physiology, 267, H1785-H1794. https://doi.org/10.1152/ajpheart.1994.267.5.H1785

[15] Depre, C., Vanoverschelde, J.L., Goudemant, J.F., Mottet, I. and Hue, L. (1995) Protection against Ischemic Injury by Nonvasoactive Concentrations of Nitric Oxide Synthase Inhibitors in the Perfused Rabbit Heart. Circulation, 92, 1911-1918. https://doi.org/10.1161/01.CIR.92.7.1911

[16] Apstein, C.S., Gravino, F.N. and Haudenschild, C.C. (1983) Determinants of a Protective Effect of Glucose and Insulin on the Ischemic Myocardium. Effects on Contractile Function, Diastolic Compliance, Metabolism, and Ultrastructure during Ischemia and Reperfusion. Circulation Research, 52, 515-526.

https://doi.org/10.1161/01.RES.52.5.515

[17] Eberli, F.R., Weinberg, E.O., Grice, W.N., Horowitz, G.L. and Apstein, C.S. (1991) Protective Effect of Increased Glycolytic Substrate against Systolic and Diastolic Dysfunction and Increased Coronary Resistance from Prolonged Global Underperfusion and Reperfusion in Isolated Rabbit Hearts Perfused with Erythrocyte Suspensions. Circulation Research, 68, 466-481.

https://doi.org/10.1161/01.RES.68.2.466

[18] King, L.M., Boucher, F. and Opie, L.H. (1995) Coronary Flow and Glucose Delivery as Determinants of Contracture in the Ischemic Myocardium. Journal of Molecular and Cellular Cardiology, 27, 701-720. https://doi.org/10.1016/S0022-2828(08)80061-2

[19] Alekseyev, M., Alekseyev, A., Dowzhikov, A. and Labin, S. (2014) Digital Analytic Cardiography (DACG), a New Method for Quantitative Tropism Assessment of the Myocardium. International Journal of Cardiovascular Research, 3, 6.

https://www.scitechnol.com/digital-analytic-cardiography-new-method-quantitativ e-trophism-assessment-myocardium-gA3N.php?article_id=2463

[20] Alekseev, M.U. and Alekseev, A.M. (2017) Differential Analysis of Nitroglycerin Influence on Myocardium in Coronary Artery Disease Patients. International Journal of Cardiovascular Research, 6, 6 .

https://www.scitechnol.com/peer-review/differential-analysis-of-nitroglycerin-influen ce-on-myocardium-in-coronary-artery-disease-patients-02mT.php?article_id=7063

[21] Opie, L. (1998) The Heart: Physiology, from Cell to Circulation. Lippincott-Raven, Philadelphia.

[22] Stanley, W.C., Lopaschuk, G.D., Hall, J.L. and McCormack, J.G. (1997) Regulation of Myocardial Carbohydrate Metabolism under Normal and Ischaemic Conditions: Potential for Pharmacological Interventions. Cardiovascular Research, 33, 243-257. https://doi.org/10.1016/S0008-6363(96)00245-3

[23] Balaban, R.S., Kantor, H.L., Katz, L.A. and Briggs, R.W. (1986) Relation between Work and Phosphate Metabolite in the in Vivo Paced Mammalian Heart. Science, 232, 1121-1123. https://doi.org/10.1126/science.3704638

[24] Wisneski, J.A., Gertz, E.W., Neese, R.A., Gruenke, L.D., Morris, D.L. and Craig, J.C. (1985) Metabolic Fate of Extracted Glucose in Normal Human Myocardium. Journal of Clinical Investigation, 76, 1819-1827. https://doi.org/10.1172/JCI112174

[25] Wisneski, J.A., Gertz, E.W., Neese, R.A. and Mayr, M. (1987) Myocardial Metabolism of Free Fatty Acids: Studies with 14C-Labeled Substrates in Humans. Journal of Clinical Investigation, 79, 359-366. https://doi.org/10.1172/JCI112820

[26] Wisneski, J.A., Stanley, W.C., Gertz, E.W. and Neese, R.A. (1990) Effects of Hyperglycemia on Myocardial Glycolytic Activity in Normal Humans. Journal of Clinical Investigation, 85, 1648-1656. https://doi.org/10.1172/JCI114616 
[27] Liedtke, A.J. (1981) Alterations in Carbohydrate and Lipid Metabolism in the Acutely Ischemic Heart. Progress in Cardiovascular Diseases, 23, 321-326. https://doi.org/10.1016/0033-0620(81)90019-0

[28] McNulty, P.H., Sinusas, A.J., Shi, C.Q., et al. (1996) Glucosemetabolismdistal to a Critical Coronary Stenosis in a Canine Model of Low-Flow Myocardialischemia. Journal of Clinical Investigation, 98, 62-69. https://doi.org/10.1172/JCI118778

[29] Bugas, G.R., Jovanovic, S., Crawford, R.M., et al. (2004) Hypoxia-Induced Preconditioning in Adult Stimulated Cardiomyocytes Is Mediated by Opening and Trafficking of Sarcolemmal $\mathrm{K}_{\mathrm{ATP}}$ Channels. The FASEB Journal, 18, 1046-1048. https://doi.org/10.1096/fj.04-1602fje

[30] Ruiz, P.E., Leblanc, N., Delorenzi, F., et al. (1992) Effects of K+-Channel Blockers on the Action Potential of Hypoxic Rabbit Myocardium. British Journal of Pharmacology, 106, 924-930. https://doi.org/10.1111/j.1476-5381.1992.tb14436.x

[31] Shah, M. and Haylett, D.G. (2000) The Pharmacology of hSK1 Ca2+-Activated K+ Channels Expressed in Mammalian Cell Lines. British Journal of Pharmacology, 129, 627-630. https://doi.org/10.1038/sj.bjp.0703111

[32] Fabiato, A. and Fabiato, F. (1978) Effects of $\mathrm{pH}$ on the Myofilaments and the Sarcoplasmic Reticulum of Skinned Cells from Cardiac and Skeletal Muscles. The Journal of Physiology, 276, 233-255. https://doi.org/10.1113/jphysiol.1978.sp012231

[33] Noakes, T.D. (1981) Exercise and the Heart. MD Thesis, University of Cape Town, Cape Town.

[34] Bricknell, O.L. and Opie, L.H. (1978) Glycolytic ATP and Its Products during Ischemia in Isolated Langendorff-Perfused Rat Hearts. Recent Advances in Studies on Cardiac Structure and Metabolism, 11, 509-519.

[35] Entman, M.L., Bornet, E.P., Van Winkle, W.B., Goldstein, M.A. and Schwartz, A. (1977) Association of Glycogenolysis with Cardiac Sarcoplasmic Reticulum. Journal of Molecular and Cellular Cardiology, 9, 515-528. https://doi.org/10.1016/S0022-2828(77)80367-2

[36] Mozaffarian, D., Benjamin, E.J., Go, A.S., Arnett, D.K., Blaha, M.J., Cushman, M., et al. (2016) Heart Disease and Stroke Statistics-2016 Update: A Report from the American Heart Association. Circulation, 133, e38-e360. https://doi.org/10.1161/CIR.0000000000000350 